\title{
Analysis of Shielding Effectiveness of Metallic Rectangular Enclosures Using Efficient Integral Equations
}

\author{
Ramin Khosravi ${ }^{1}$, Hossein Maftooli $^{2}$, Hamidreza Karami ${ }^{2, *}$ \\ ${ }^{1}$ Department of Electrical, Computer and Biomedical Engineering, Qazvin Branch, Islamic Azad University, Qazvin, Iran \\ ${ }^{2}$ Department of Electrical Engineering, Amirkabir University of Technology, Tehran, Iran \\ *Corresponding Author: hamidr_karami@aut.ac.ir, Hamidreza.karami@epfl.ch
}

Copyright @ 2013 Horizon Research Publishing All rights reserved.

\begin{abstract}
The paper proposes an efficient modeling technique for electromagnetic analysis of a rectangular metallic enclosure with multiple apertures, using the surface equivalent principle and frequency-domain integral equations. The surface equivalence principle is used to replace the apertures by a prefect electrically conducting surfaces while introducing appropriate magnetic current densities on both sides of it with opposite sign. As a result, the problem is reduced to two independent regions. The first region is outside the enclosure (exterior problem) where the free space Green's function with the aid of image theory can be used to obtain the total fields in it. The second region is the interior of the enclosure (interior problem) that a closed-form expression for its dyadic Green's function is available. Finally the governing frequency-domain magnetic field integral equation for the structure is derived by enforcing the continuity of the tangential magnetic field at the apertures. To solve the resulting integral equations, method of moments is used. The validity of the proposed method is confirmed by comparing the simulation results of several case studies with those obtained using the commercial MWS-CST finite integration code.
\end{abstract}

Keywords Shielding Effectiveness, Metallic Rectangular Enclosure, Electromagnetic Compatibility, Method of Moments, Dyadic Green's Function

\section{Introduction}

In practice, to protect sensitive electric and electronic devices from intentional or unintentional electromagnetic interference (EMI), metallic enclosures are commonly used. Apertures and slots on the enclosure surfaces which are necessary for visibility, ventilation purpose and input/output cable access may degrade the shielding effectiveness (SE).

Several analytical and numerical methods have been proposed for calculating the SE of perforated metallic enclosures. Analytical methods are restricted to very simple geometries with some approximations [1], [2]. On the other hand, conventional numerical methods like finite-element method [3], finite-difference time-domain method [4], method of moments [5]-[7], transmission-line method [8], and hybrid methods [9] can be applied for analyzing shielding enclosures with arbitrary geometry by desired accuracy at the cost of CPU time and memory resources.

For metallic rectangular enclosures with homogenous media integral equation based solvers appear to be more suitable and versatile as they only need to discretize the surface rather than volume and implicitly impose the radiation conditions.

In this paper, we have proposed an efficient method for analyzing a metallic rectangular enclosure with arbitrary shaped apertures. In this method, the surface equivalence principle [10] is first applied to replace the apertures with the PEC surfaces and equivalent magnetic current densities on their both sides with opposite sign. As a result, the problem of the metallic rectangular is reduced to a closed cavity with a half-space region on top of it [6]. A closed-form expression for the frequency-domain dyadic Green's function of the closed cavity is available. Having obtained the closed-form of the dyadic Green's function, the governing frequency-domain magnetic field integral equations for the metallic enclosure structure are derived by enforcing the continuity of the tangential magnetic field at the aperture interfaces. Finally, the method of moments (MoM) scheme is used for solving the resultant frequency-domain magnetic field integral equations system [11].

The organization of the paper is as follows. Section II describes the proposed method for analyzing metallic rectangular enclosure with arbitrary shaped apertures. The derivation of the governing MFIEs in this Section is followed by an outline of the solution methodology and numerical considerations. In Section III, the validity of the proposed method will be confirmed by comparing the simulation results of several case studies with those obtained using the commercial MWS-CST [14] finite integration code. 


\section{Theory}

Consider a metallic rectangular enclosure with an arbitrary shaped aperture on its top wall located in free-space, as shown in Fig. 1. The dimensions of the cavity are $a \times b \times c$. With reference to Fig. 1, the permittivity, permeability and conductivity of dielectric in metallic cavity are $\varepsilon_{2}, \mu_{2}$ and $\sigma_{2}$, respectively.

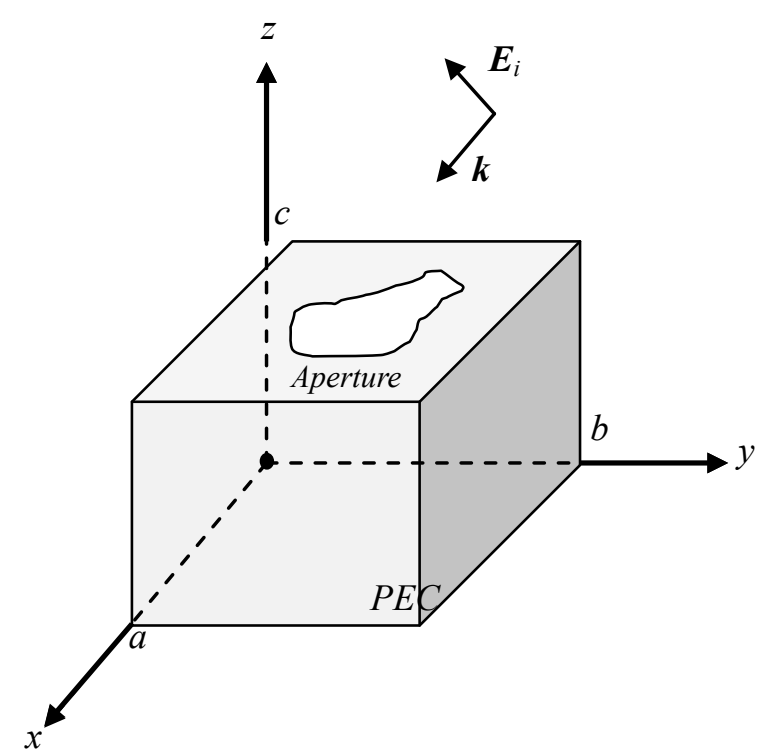

Figure 1. Geometry of a metallic rectangular enclosure with arbitrarily shaped aperture illuminated by an arbitrarily polarized plane wave at oblique incidence

It is assume that the aperture is far enough away from the cavity walls so that the image theory could be applied. Using the surface equivalence principle, the aperture is covered by PEC planes. It is well known that the electric current density cannot radiate in the vicinity of a PEC surface [10], thereby only the equivalent magnetic current density over the aperture radiate in half-space $(\boldsymbol{M}(\boldsymbol{r}))$ and rectangular cavity $(-M(r))$ regions and the electric current densities are ignored, as shown in Fig. 2.

By enforcing the continuity of the tangential magnetic field over the aperture, (1) can be obtained as follows,

$$
\begin{array}{r}
\boldsymbol{H}_{\mathrm{tan}}^{1}(\boldsymbol{M}(\boldsymbol{r}))+\boldsymbol{H}_{\mathrm{tan}}^{\text {out }}(\text { sources })= \\
\boldsymbol{H}_{\mathrm{tan}}^{2}(-\boldsymbol{M}(\boldsymbol{r}))+\boldsymbol{H}_{\mathrm{tan}}^{\text {in }}(\text { sources })
\end{array}
$$

where $\boldsymbol{H}_{\tan }{ }^{1}$ and $\boldsymbol{H}_{\tan }{ }^{2}$ represent the tangential components of. magnetic fields due to the equivalent magnetic current density, $\boldsymbol{M}$, radiated into the half-space and cavity regions, respectively. $\mathrm{H}_{\tan }$ out and $\mathrm{H}_{\tan }$ in represent the tangential magnetic fields due to the sources located in free space and metallic cavity, respectively. The $\boldsymbol{r}$ denotes the observation vector.

Using the mixed potential integral equation formulation [11], the tangential components of frequency-domain half-space magnetic fields can be obtained as follows,

$$
\begin{aligned}
\boldsymbol{H}_{\mathrm{tan}}^{1} & (\boldsymbol{M}(\boldsymbol{r}))= \\
& \left\lfloor-j \omega \boldsymbol{F}(\boldsymbol{M}(\boldsymbol{r}))-\nabla \phi_{m}(\boldsymbol{M}(\boldsymbol{r}))\right\rfloor_{\tan }
\end{aligned}
$$

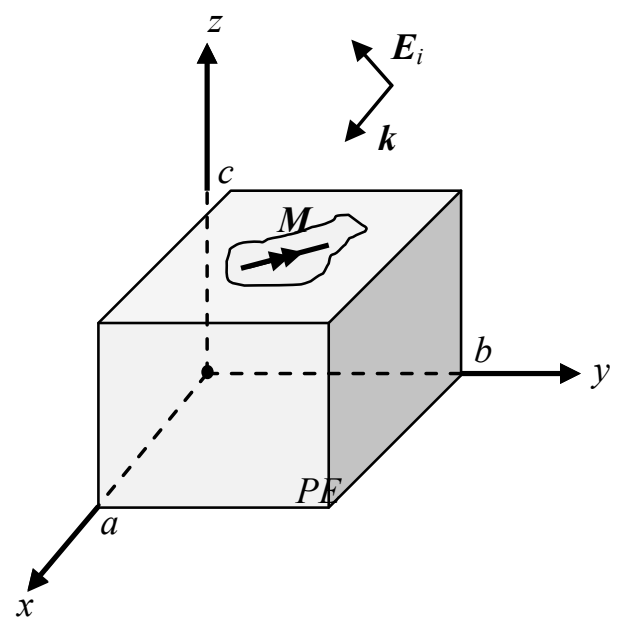

Figure 2. Geometry of equivalent problem as shown in Figure 1 with arbitrarily shaped aperture replaced by an equivalent magnetic current density

where $\boldsymbol{F}$ and $\varphi_{\mathrm{m}}$ are, respectively, the electric vector potential and magnetic scalar potential in frequency-domain, whose expressions for half-space can be written as follows,

$$
\begin{gathered}
F(\boldsymbol{M}(\boldsymbol{r}))=\frac{\varepsilon_{1}}{2 \pi} \int_{S} \frac{\boldsymbol{M}\left(\boldsymbol{r}^{\prime}\right)}{R} e^{-j k R} d s^{\prime} \\
\phi_{m}(\boldsymbol{M}(\boldsymbol{r}))=-\frac{1}{j \omega 2 \pi \mu_{1}} \int_{S} \frac{\nabla^{\prime} \cdot \boldsymbol{M}\left(\boldsymbol{r}^{\prime}\right)}{R} e^{-j k R} d s^{\prime}
\end{gathered}
$$

and for cavity region,

$$
\begin{aligned}
& \boldsymbol{H}_{\mathrm{tan}}^{2}(\boldsymbol{M}(\boldsymbol{r}))=-j \omega\left(\varepsilon_{2}-j \sigma / \omega\right) \\
& \int_{S}^{\overline{\boldsymbol{G}}_{e 2}\left(\boldsymbol{r}, \boldsymbol{r}^{\prime}\right) \cdot \boldsymbol{M}\left(\boldsymbol{r}^{\prime}\right) d s^{\prime}}
\end{aligned}
$$

According to the method of scattering superposition [12], the dyadic Green's function $\overline{\bar{G}}_{e 2}\left(r, r^{\prime}\right)$ for the rectangular cavity can be obtained as the sum of unbounded and the scattering green dyadic, i.e.

$$
\left.\underline{\overline{\bar{G}}}_{e 2}\left(\boldsymbol{r}, \boldsymbol{r}^{\prime}\right)=\overline{\overline{\boldsymbol{G}}}_{e 2 s} \underline{\underline{\underline{\boldsymbol{G}}}}, \boldsymbol{r}^{\prime}\right)+\overline{\overline{\boldsymbol{G}}}_{e 2 o}\left(\boldsymbol{r}, \boldsymbol{r}^{\prime}\right)
$$

where $\overline{\overline{\boldsymbol{G}}}_{e 2 o}\left(\boldsymbol{r}, \boldsymbol{r}^{\prime}\right)$ and $\overline{\boldsymbol{G}}_{e 2 s}\left(\boldsymbol{r}, \boldsymbol{r}^{\prime}\right)$ are the unbounded and the scattering Green dyads, respectively. For a rectangular cavity the general expressions for the scattering and unbounded dyads has been obtained in [13]. In the most general case $\overline{\bar{G}}_{e 2}\left(r, r^{\prime}\right)$ has nine components while as we are only concern with transverse components of fields due to sources having no longitudinal components, these nine components reduced to only 4 components, i.e., $G_{\mathrm{ij}}(i, j=x, y)$ where $G_{\mathrm{ij}}$ is the $i$-th component of the field due to a unit j-directed current source $\delta\left(\boldsymbol{r}, \boldsymbol{r}^{\prime}\right) \hat{\boldsymbol{J}}$. These four components for a rectangular cavity with length $a$, width $b$ and short circuited planes at $z_{1}=-d, z_{2}=0$ can be cast in compact form as (6). 


$$
\begin{aligned}
& {\left[\overline{\overline{\boldsymbol{G}}}_{e 2}\left(\boldsymbol{r}, \boldsymbol{r}^{\prime} ; t^{\prime}\right)\right]_{\tan }=-\frac{2}{a b} \sum_{m=0}^{+\infty} \sum_{n=0}^{+\infty} \frac{2-\delta_{m n}}{k_{g} k_{c}^{2} \sin \left(k_{g} d\right)}} \\
& {\left[\begin{array}{l}
\boldsymbol{m}_{o}(\boldsymbol{r}) \boldsymbol{m}_{o}\left(\boldsymbol{r}^{\prime}\right) \\
+\boldsymbol{n}_{e}(\boldsymbol{r}) \boldsymbol{n}_{e}\left(\boldsymbol{r}^{\prime}\right)
\end{array}\right] \cos \left(\begin{array}{c}
z \\
k_{g} \\
z^{\prime}
\end{array}\right) \cos \left(k _ { g } \left(\begin{array}{l}
\left.\left.z^{\prime}+d\right)\right) z^{>} z^{\prime} \\
z
\end{array}\right.\right.} \\
& \delta_{i}=\left\{\begin{array}{ll}
0 & i \neq 0 \\
1 & i=0
\end{array}, k_{g}=\sqrt{k^{2}-k_{c}^{2}}, k_{c}=\sqrt{k_{x}^{2}+k_{y}^{2}}\right. \\
& \boldsymbol{m}_{o}(\boldsymbol{r})=k_{y} S_{x} C_{y} \hat{\boldsymbol{x}}-k_{x} C_{x} S_{y} \hat{\boldsymbol{y}} \\
& \boldsymbol{n}_{e}(\boldsymbol{r})=\frac{k_{g}}{k} k_{x} S_{x} C_{y} \hat{\boldsymbol{x}}+\frac{k_{g}}{k} k_{y} C_{x} S_{y} \hat{\boldsymbol{y}} \\
& k_{x}=\frac{m \pi}{a}, k_{y}=\frac{n \pi}{b}, S_{x}=\sin \left(k_{x} x\right), C_{y}=\cos \left(k_{y} y\right) \\
& S_{y}=\sin \left(k_{y} y\right), C_{x}=\cos \left(k_{x} x\right)
\end{aligned}
$$

where the top line applies to $z \geq z^{\prime}$ and the bottom line is for $z \leq z^{\prime}$ and $\mathrm{k}=\omega(\mu(\varepsilon-\mathrm{j} \sigma / \omega))^{0.5}$.

Finally to numerically solve (1), $\boldsymbol{M}(\boldsymbol{r})$ on the aperture is expanded using $N_{\mathrm{s}}$ spatial basis function as,

$$
\boldsymbol{M}(\boldsymbol{r})=\sum_{n=1}^{N_{s}} K_{n} \boldsymbol{f}_{n}(\boldsymbol{r})
$$

where $K_{\mathrm{n}}$ is the unknown expansion coefficient and $\boldsymbol{f}_{\mathrm{n}}$ is the spatial basis function. In this paper, the spatial basis function is assumed to be roof-top [13]. In the case of enclosures with multiple apertures, each aperture is replaced with appropriate magnetic currents and the same procedure can be applied.

By substituting (2) and (4) in (1) and multiplying both sides of the resulting equation with $f_{\mathrm{m}}$ and then integration over the space (i.e., by Galerkin testing), $K_{\mathrm{n}}$ can be obtained from the linear system of equation (8),

$$
\mathbf{Y K}=\mathbf{I}^{\text {exc }}
$$

where the structure of the admittance matrix, $\mathbf{Y}$, and vectors $\mathbf{I}^{\mathrm{exc}}$ and $\mathbf{K}$ are shown in (9).

$$
\left(\begin{array}{cc}
\mathbf{Y}^{x x} & \mathbf{Y}^{x y} \\
\mathbf{Y}^{y x} & \mathbf{Y}^{y y}
\end{array}\right)\left(\begin{array}{l}
\mathbf{K}^{x} \\
\mathbf{K}^{y}
\end{array}\right)=\left(\begin{array}{l}
\mathbf{I}^{e x c, x} \\
\mathbf{I}^{e x c, y}
\end{array}\right)
$$

It is worth to mention that the admittance matrix $\mathbf{Y}$ accounts the mutual coupling effects in the case of multiple apertures in a rigorous full wave manner. The entries of vector $\mathbf{I}^{\text {exc }}$ and admittance matrix $\mathbf{Y}$ are given in (10), (11),

$$
I^{e x c, u}=-\int_{S_{m}}\left(\boldsymbol{h}_{\mathrm{tan}}^{\text {out }}-\boldsymbol{h}_{\mathrm{tan}}^{i n}\right) \cdot \hat{\boldsymbol{a}}_{u} f_{m}^{u} d s, u=x, y
$$

where $f_{m}^{u}(u=x, y)$ denotes the $u$ components of the rooftop basis function and the admittance matrix $\mathbf{Y}$ can be split in two independent parts, one part accounts for the free space green function and the other related to cavity green function. The elements of these matrices are shown in (11),

$$
\begin{aligned}
& \mathbf{Y}=\mathbf{Y}^{\text {FreeSpace }}+\mathbf{Y}^{\text {Cavity }} \\
& \mathbf{Y}^{\text {FreeSpace }}=\left[\begin{array}{ll}
\mathbf{Y}^{x x_{-} \text {FreeSpace }} & \mathbf{Y}^{x y_{-} \text {FreeSpace }} \\
\mathbf{Y}^{y x_{-} \text {FreeSpace }} & \mathbf{Y}^{y y_{-} \text {FreeSpace }}
\end{array}\right] \\
& \mathbf{Y}^{\text {Cavity }}=\left[\begin{array}{ll}
\mathbf{Y}^{x x_{-} \text {Cavity }} & \mathbf{Y}^{x y_{-} \text {Cavity }} \\
\mathbf{Y}^{y x_{-} \text {Cavity }} & \mathbf{Y}^{y y_{-} \text {Cavity }}
\end{array}\right] \\
& \mathbf{Y}_{j-\bar{j}^{\prime}}^{u v \text { FreeSpace }}=-\hat{\boldsymbol{a}}_{u} \cdot \hat{\boldsymbol{a}}_{v} \frac{j \omega \varepsilon_{1}}{2 \pi} \int_{S_{m}} \int_{S_{n}}\left(f_{m}^{u} \frac{e^{-j k R}}{R} f_{n}^{v}\right) d s^{\prime} d s \\
& -\frac{1}{j \omega 2 \pi \mu_{1}} \int_{S_{m}} \int_{S_{n}}\left(\frac{\partial f_{m}^{u}}{\partial u} \frac{e^{-j k R}}{R} \frac{\partial f_{n}^{v}}{\partial v}\right) d s^{\prime} d s, u, v=x, y \\
& \mathbf{Y}^{u v_{-} \text {Cavity }}=\sum_{m, n=0}^{+\infty} Q_{m n}^{u v} \int_{S_{m}} \int_{S_{n}}\left(f_{m}^{u} g_{u} g_{v} f_{n}^{v}\right) d s^{\prime} d s, u, v=x, y
\end{aligned}
$$

where $g_{x}=S_{x} C_{y}, g_{y}=C_{x} S_{y}$ and the expressions for the scalars $Q_{m n}^{u v}$ and $C t e$ are as follows,

$$
\begin{aligned}
& C t e=j \omega\left(\varepsilon_{2}-j \sigma_{2} / \omega\right) \frac{2}{a b} \frac{2-\delta_{m n}}{k_{g} k_{c}^{2} \tan \left(k_{g} d\right)} \\
& Q_{m n}^{x x}=C t e\left(\left(\frac{k_{g}}{k}\right)^{2} k_{x}^{2}+k_{y}^{2}\right) \\
& Q_{m n}^{y y}=C t e\left(\left(\frac{k_{g}}{k}\right)^{2} k_{y}^{2}+k_{x}^{2}\right) \\
& Q_{m n}^{x y}=Q_{m n}^{y x}=C t e\left(\left(\frac{k_{g}}{k}\right)^{2} k_{x} k_{y}-k_{x} k_{y}\right)
\end{aligned}
$$

It should be noted that for ease of presentation here it is assumed that the cavity walls have negligible thickness. However the proposed method can easily handle situations with finite wall thickness, it just needs to consider two sets of magnetic currents at both sides of the aperture, i.e., the aperture can be replaced with a rectangular cavity with appropriate magnetic currents located over two sides of it.

\section{Numerical Results}

To validate the proposed method, we have analyzed several case studies. For brevity, we present only the shielding effectiveness related to a metallic enclosure with a) a single aperture, b) multiple apertures. The shielding effectiveness can be defined as the ratio of the electric field at a given point inside the enclosure to the field at the same point in the absence of the enclosure.

\subsection{A Metallic Enclosure with a Single Aperture}

The first example consists of a metallic rectangular enclosure with dimensions $30 \mathrm{~cm} \times 12 \mathrm{~cm} \times 30 \mathrm{~cm}$ and with a rectangular aperture $(18 \mathrm{~cm} \times 5 \mathrm{~cm})$ placed at the center of the front side as shown in the inset of Fig. 3. The calculated SE-y (SE for the y-component of electric field) at the center of 
enclosure $(x, y, z)=(15,6,15) \mathrm{cm}$ is compared with the simulation results obtained by MWS-CST code [14] based on the time-domain finite integration technique. To consider the infinite flange at front of the enclosure, all examples are modeled in MWS-CST using decoupling plate. For MWS-CST simulation, accuracy of the energy convergence, number of meshcells and mesh per wavelength are $-80 \mathrm{~dB}$, 24552 and 40 respectively. As shown in Fig. 3, a good agreement is observed between the results. Without loss of the generality normal plane wave incident is considered with the electric field polarization along the $y$ axis. For this example the number of roof-top basis function is 399 . The number of total modes in constructing the cavity green function is 3600 .

In the second example the aperture specific earlier (inset of Fig. 3) is replaced by a rectangular slot $(10 \mathrm{~cm} \times 0.5 \mathrm{~cm})$ which located at the front side. The calculated SE-y at the center of enclosure is compared with the simulation results obtained by MWS-CST. As can be seen in Fig. 4, a good agreement is observed between the results. The number of roof-top basis functions is 201 . The total number of the used modes is 8100 .

\subsection{A Metallic Enclosure with Multiple Apertures}

In inset of Fig. 5, an array of four apertures in front side of the previous enclosure is shown. The magnetic currents on the apertures are expanded with 556 Roof-Top basis functions. The number of modes for calculating the cavity green function is 10000 in this simulation. A comparison between the proposed method and MWS-CST result, as shown in Fig. 5, indicates a good agreement between them.

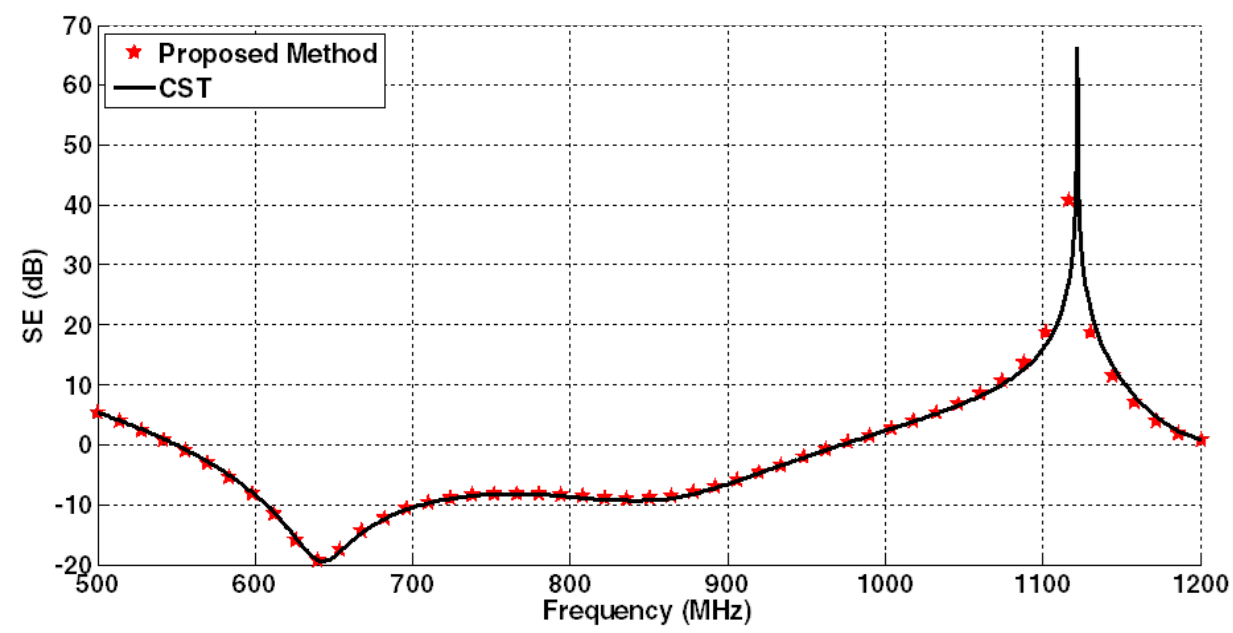

Figure 3. Comparison between SE-y of a rectangular enclosure calculated by proposed method (stars) and MWS-CST (solid line) with L=18cm, $w=5 \mathrm{~cm}$

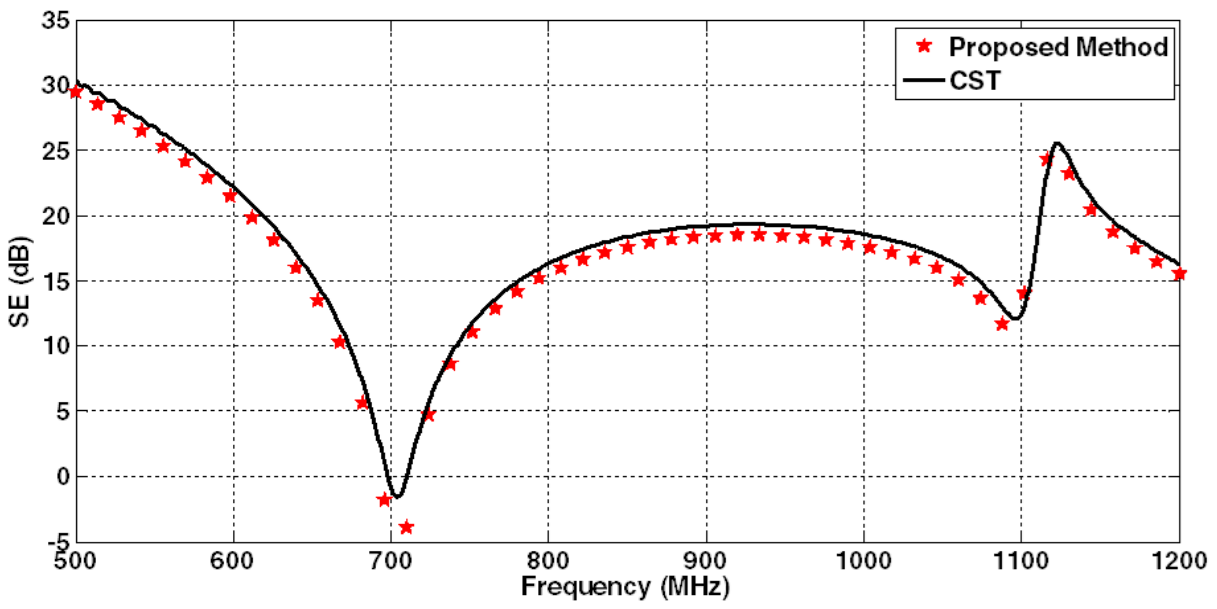

Figure 4. Comparison between SE-y of a rectangular enclosure calculated with proposed method (stars) and MWS-CST (solid line) with L=10cm, $\mathrm{w}=0.5 \mathrm{~cm}$ 


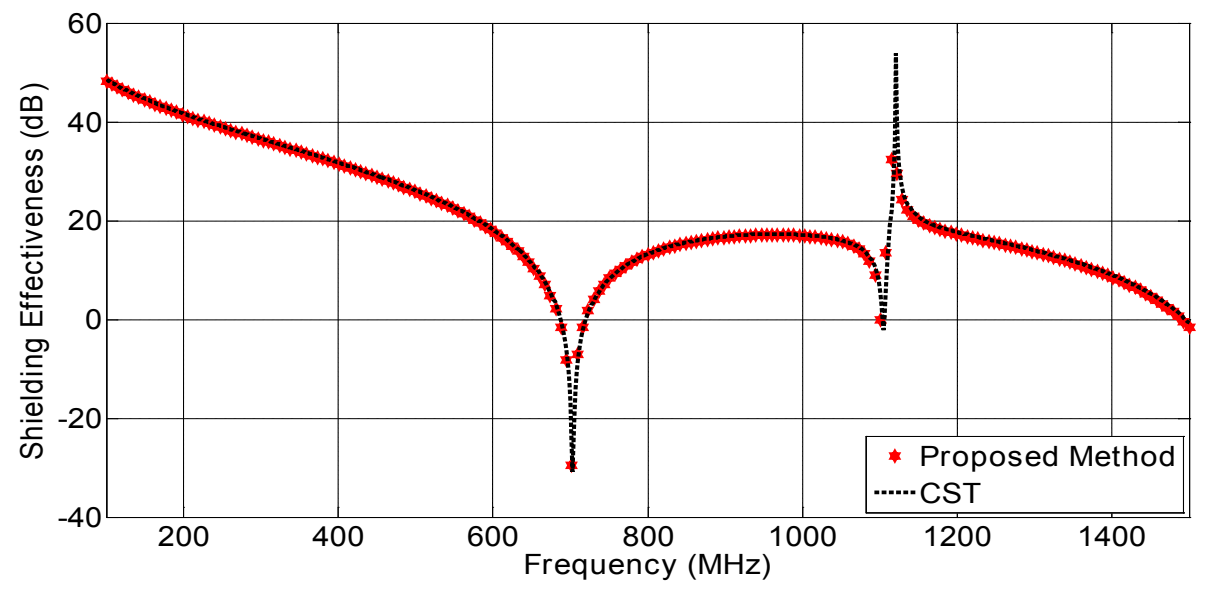

Figure 5. Comparison between the electric SE-y of a rectangular enclosure with an array of apertures calculated with proposed method (stars) and MWS-CST (solid line)

\section{Conclusion}

This paper described an efficient frequency-domain integral equation based analysis for rectangular enclosures with multiple apertures in a rigorous full wave manner which accounts the mutual coupling effects between them. Using the surface equivalence principle the original problem is divided into two independent ones which related to each other via the introduced magnetic currents on the common apertures. Here it is assumed that the apertures are far enough away from the edges so that the image theory could be applied. In addition, the edge-diffracted fields are neglected. However it should be noted that for enclosures with finite wall front the proposed method can be modified to overcome this limitation, i.e. it can be accomplished by considering the radiation of the electric current on the PEC wall front. The governing magnetic field integral equation is constructed by enforcing the continuity of the tangential magnetic fields at the common apertures. To solve the resulting integral equation a method of moments is used. The proposed method benefits the commercial numerical codes as the only unknowns of the problem are the magnetic currents at the apertures. The validity of the proposed method is confirmed by comparing the simulation results of several case studies with those obtained using the commercial MWS-CST finite integration code.

\section{REFERENCES}

[1] H. A. Bethe, "Theory of diffraction by small holes," Phys. Rev., 2nd series, vol. 66, pp. 163-182, 1944.

[2] M. P. Robinson, T.M. Benson, C. Christopoulos, J. F. Dawson,M. D. Ganley, A. C. Marvin, S. J. Porter, and D. W. P. Thomas, "Analytical formulation for the shielding effectiveness of enclosures with apertures," IEEE Trans. Electromagn. Compat., vol. 40, no. 3, pp. 240-248, Aug. 1998.

[3] S. Benhassine, L. Pinchon, and W. Tabbara, "An efficient finite-element time-domain method for the analysis of the coupling between wave and shielded enclosure," IEEE Trans. Magn., vol. 38, no. 2, pp. 709-712, Mar. 2002.

[4] M. Li, J. Nuebel, J. L. Drewniak, T. H. Hubing, R. E. DuBroff, and T. P. Van Doren, "EMI from cavity modes of shielding enclosures-FDTD modeling and measurements," IEEE Trans. Electromagn.Compat., vol. 42, no. 1, pp. 29-38, Feb. 2000.

[5] R. Araneo and G. Lovat, "Fast MoM analysis of the shielding effectiveness of rectangular enclosures with apertures, metal plates, and conducting objects," IEEE Trans. Electromag. Compat., vol. 51, no. 2, pp. 274-283, May 2009.

[6] P. Dehkhoda, A. Tavakoli, and M. Azadifar, "Shielding effectiveness of an enclosure with finite wall thickness and perforated opposing walls at oblique incidence and arbitrary polarization by GMMoM." IEEE Trans. Electromag. Compat., vol. 54, no. 4, pp. 792-805, Aug 2012.

[7] Electromagnetic field penetration studies (NASA/CR-2000-210 297), M. D. Deshpande (2000, June). [Online]. Available: http://ntrs.nasa.gov/

[8] P. Argus, P. Fischer, A. Konrad, and A. J. Schwab, "Efficient modeling of apertures in thin conducting screens by the TLM method," in Proc. IEEE Int. Symp. Electromagn. Compat., Aug. 21-25, 2000, vol. 1, pp. 101-106.

[9] M. S. Tharf and G. I. Costache, "A hybrid finite element-Analytical solutions for in-homogeneously filled shielding enclosures," IEEE Trans. Electromagn. Compat., vol. 36, no. 4, pp. 380-385, Nov. 1994.

[10] R. F. Harrington, Time-Harmonic Electromagnetic Fields. Piscataway, NJ: IEEE Press, 2001.

[11] R. F. Harrington, Field Computation by the Moment Methods. New York: Wiley, 1993.

[12] C-T. Tai, Dyadic Green's Function in Electromagnetic Theory, IEEE Press, New York, 1994.

[13] H. Maftooli, H. R. Karami, S. H. H. Sadeghi, and R. Moini, "Output Signal Prediction of an Open-Ended Coaxial Probe When Scanning Arbitrary-Shape Surface Cracks in Metals," IEEE Trans. Instrum. Meas, vol. 61, no. 9, pp. 2384-2391,Sep. 2012.

[14] Microwave Studio, Computer Simulation Technology (CST). (2011). [Online]. Available: www.cst.de 